\title{
La tuberculose - point de départ de la chirurgie thoracique
}

par André P. Naef

\section{RÉSUMÉ}

Le développement foudroyant de la chirurgie thoracique est dû̀ à la demande extraordinaire d'interventions chirugicales dans le traitement de maladies fréquentes, la tuberculose, les valvulopathies rhumastismales et la maladie coronarienne. L'épidémie de la tuberculose en fut le point de départ. Dès 1939 les jeunes chirurgiens formés dans les sanatoria furent confrontés à la chirurgie thoracique de guerre. C'est ainsi qu'ils se familiarisèrent avec les plaies du cœur et des grands vaisseaux. De retour à la vie civile il deviendront les pionniers de la chirurgie cardiaque, d'abord primitive et à l'aveugle. En 40 ans cette chirurgie deviendra la discipline pluri-disciplinaire sophistiquée d'aujourd'hui, couronnée par la greffe du cœur devenue une opération de routine.

Au début de ce bref exposé j’aimerais préciser que je ne suis pas un historien de formation mais que j'appartiens à la génération de chirurgiens qui a eu la chance de vivre tout le développement explosif de la chirurgie du thorax de 1940 à nos jours. Par conséquent, les souvenirs que j'ai rassemblés dans deux livres publiés récemment ${ }^{1,2}$ ne sont pas le fruit d'un travail d'historien mais le récit authentique d'un contemporain ayant participé à la naissance d'une nouvelle discipline chirurgicale, la chirurgie thoracique et cardio-vasculaire. Mes récits d'actualité et ceux de mes contemporains fourniront sans doute le matériel qui permettra à l'avenir d'écrire une histoire professionnelle de la médecine thoracique. Dans ma rétrospective d'aujourd'hui j'aimerais démontrer le rôle clé joué par la tuberculose dans la naissance de notre discipline.

Tout le monde est d'accord que les progrès de la médecine sont en général dus à un ensemble d'événements concomitants, dans le cas particulier l'introduction de la narcose intra-trachéale et du cathétérisme cardiaque, de même que la découverte de l'héparine, des antibiotiques et de la ciclosporine. Mais la rapidité spectaculaire, de moins d'un demi-siècle, avec laquelle la chirurgie thoracique a vu le jour, est liée à ce que l'on pourrait appeler «la loi 
du marché». J'entends par là la demande extraordinaire d'interventions chirurgicales dans le traitement de maladies fréquentes, presque épidémiques, la tuberculose, les valvulopathies rhumatismales et les coronaropathies qui ont motivé et formé un grand nombre de chirurgiens, d'abord non spécialisés, puis de plus en plus axés sur une nouvelle discipline. La tuberculose fut la dernière grande épidémie avant le SIDA et elle a joué le rôle déterminant dans cette évolution. Par conséquent, nous, les chirurgiens des années cinquante, avons-nous encore été les témoins d'une maladie incurable, presque oubliée aujourd'hui, et d'une chirurgie primitive mais héroïque qu'on a de la peine à se représenter de nos jours.

C'est le tandem malade tuberculeux et médecin phtisiologue qui est à l'origine de la pneumologie moderne, et pour les premiers chirurgiens thoraciques les tuberculeux incurables représentaient le gros de leur clientèle. En fait, avant 1944, arrivée de la streptomycine, il n'y avait pas de traitement spécifique d'une maladie hautement contagieuse, qui avait fait des ravages, surtout depuis l'urbanisation et la prolétarisation du début de l'ère industrielle et, plus proche de nous, suite à la misère des deux guerres mondiales.

A vrai dire, cette maladie fatalement progressive avait stimulé l'esprit inventif des médecins depuis et même avant Hippocrate, qui préconisèrent d'innombrables régimes à base d'herbes, de lait de chèvre et du bon air de la mer, de la montagne ou du désert. On attribue en fait à Galien d'avoir établi le premier «sanatorium» sur un de ses domaines dans les collines dominant la baie de Naples. Dix-huit siècles plus tard, lorsqu'en 1941 j'étais interne au Sanatorium Valaisan, la cure de repos (Liegekur ou bed-rest) était encore la seule chance de guérison! Avant d'en arriver à la chirurgie, ce furent les grands médecins qui jouèrent le rôle décisif: Laennec pour le diagnostic ${ }^{3}$, Koch pour l'étiologie spécifique, Brehmer en Allemagne, Trudeau à Saranac Lake, Spengler à Davos ${ }^{4}$ pour la cure sanatoriale. La suite logique du concept de la cure de repos fut la mise au repos sélectif du poumon par le pneumothorax, introduit par Forlanini en $1882^{5}$. L'attitude fataliste et morbide de cette vie sanatoriale, que j'ai encore vécue, est la toile de fond du célèbre roman «Der Zauberberg» de Thomas Mann ${ }^{6}$.

Les chirurgiens, ou plus souvent les médecins à tempérament chirurgical - souvent eux-mêmes échoués dans ces villages de montagne du fait de leur propre maladie - s'étaient mis à poursuivre le principe de la mise au repos du poumon par collapsothérapie chirurgicale. Dans un premier temps certains, entre autres notre de Cérenville, avaient drainé les cavernes tuberculeuses à la peau, une méthode popularisée plus tard par Monaldi ${ }^{7}$. 
L'idée d'affaisser les cavernes par la résection des côtes recouvrant la zone malade a été émise simultanément à divers endroits. Nous autres Romands l'attribuons à Edouard de Cérenville, qui en parle dans sa publication en $1886^{8}$. Ce n'était pas encore la «thoracoplastie extrapleurale», terme créé par Carl Spengler de Davos en 1890. Ludolph Brauer ${ }^{9}$, éminent phtisiologue et partisan du pneumothorax, réalisa le premier que la thoracoplastie, pour être efficace, devait ressembler au pneumothorax, c'est-à-dire obtenir un réel collapsus du territoire pulmonaire atteint. Il recommanda à son chirurgien Friedrich, de Marburg, de réséquer de longs segments de la première à la onzième côte. Cette «totale Thorakoplastik», inutilement étendue en hauteur mais pas assez horizontalement, fut rendue mieux adaptée aux lésions habituellement apicales par Sauerbruch, élève de Friedrich. Sauerbruch établit sa renommée de premier chirurgien thoracique par cette opération, dont la mortalité fut encore effrayante au départ ${ }^{10}$.

Il convient aussi de rappeler aux anesthésistes, qui ont réinventé le «bloc intercostal» pour atténuer la douleur postopératoire, que Sauerbruch, comme tout le monde alors, pratiquait ses opérations en anesthésie locale! Avant l'ère de la streptomycine il fallait en effet que le malade soit conscient et puisse tousser et cracher pendant l'opération afin d'éviter la redoutable dissémination bronchogène contro-latérale. Lors de mon internat en chirurgie en 1943 notre première tâche était d'apprendre à faire l'anesthésie locale pour les thoracoplasties du patron! Alfred Brunner, l'élève de Sauerbruch et professeur à Zurich (1941-1962), faisait même ses premières lobectomies en anesthésie locale. Pour ma part, suivant mon maître, j’opérais mes premiers patients sur la table d'Overholt, en décubitus ventral, afin de faciliter le drainage trachéobronchique et prévenir cette dissémination controlatérale ${ }^{11}$.

En parlant du risque des expectorations positives abondantes, rappelons que les malades tuberculeux étaient redoutés dans les hôpitaux de ville qui, généralement, refusaient de les admettre ou les isolaient dans des pavillons sans installations chirurgicales. Ceci conduisit à l'habitude de la chirurgie itinérante. On installa des salles d'opération dans les sanatoria et au lieu de transférer les malades, c'était les chirurgiens de plaine qui se déplaçaient régulièrement pour des séances opératoires de plusieurs thoracoplasties ou lobectomies groupées. Brunner montait de Zurich à Davos, César Roux et son gendre Léon Picot de Lausanne à Leysin. Bérard de Lyon faisait de même en France et Overholt, que j'accompagnais souvent pour l'assister, se déplaçait de Boston dans les sanatoria du New Hampshire. Une conséquence 
historique de cette ségrégation des malades contagieux fut la fondation en 1884 du célèbre Brompton Hospital, berceau de la médecine thoracique britannique. Un notaire, David Rose, ayant été incapable de faire admettre un de ses employés atteint de tuberculose dans un des hôpitaux de Londres, prit l'initiative de fonder cet hôpital pour tuberculeux et obtint le patronage de la reine Victoria.

La majorité des opérés des premiers chirurgiens thoraciques se recrutait donc parmi les malades tuberculeux. Cette relation allait encore s'accentuer avec le «boom» de l'exérèse pulmonaire des années cinquante! $90 \%$ de mes premières lobectomies - comme celles d'Overholt, Brunner, Bérard et beaucoup d'autres chirurgiens - étaient pratiquées pour tuberculose pulmonaire. Cette brève histoire de l'exérèse pour tuberculose pulmonaire, une véritable «aventure», représente le trait d'union entre l'époque primitive des thoracoplasties de Sauerbruch et la chirurgie cardiaque sophistiquée de notre temps.

Ouvrant une parenthèse, il convient de rappeler la découverte de la streptomycine en 1944 par Selman Abraham Waksman. Avec la pénicilline c'est probablement le médicament qui a le plus contribué aux progrès de la chirurgie en général et de la chirurgie thoracique en particulier. Il n'y a que l'éther, cent ans plus tôt, et la ciclosporine, trois décennies plus tard, qui aient joué un rôle comparable.

Pour en revenir aux débuts de l'exérèse pulmonaire il faut remonter à l'année 1881 et à la tragique histoire du Dr. Block, de Danzig, qui fut le premier à tenter une résection pulmonaire chez l'humain. Dans une lettre au «Boston Medical and Surgical Journal» (1883), un Américain, G. L. Walton ${ }^{12}$, raconte avoir rencontré Block en visite dans le laboratoire de Virchow en 1882, démontrant l'aisance avec laquelle il pouvait pratiquer des lobectomies chez des animaux de laboratoire (lapins, chiens, etc.), animaux qui récupéraient rapidement et complètement de ces interventions. Walton remarque cependant que Block ne parvint guère à transmettre son enthousiasme à l'assistance qui doutait de l'innocuité de ces interventions chez d'éventuels patients humains. Block avait demandé à Walton de traduire un manuscrit pour le Journal de Boston, mais de retour à Berlin après ses vacances d'été Walton apprit l'histoire de la mort tragique de Block: apparemment il entreprit d'opérer une patiente atteinte d'une tuberculose et qui, moins résistante que les lapins, mourut pendant ou immédiatement après l'opération. Désespéré et menacé de poursuites judiciaires, le chirurgien se suicida, "shooting himself through the head». Voilà pour le témoignage de Walton. Meade ${ }^{13}$, qui rapporte d'ailleurs que Block avait pratiqué une 
résection bilatérale et que l'autopsie ne révéla pas de tuberculose (ce qui ne ressort pas de la lettre de Walton) essaya d'en savoir davantage. Il questionna Nissen, qui devait être assez bien au courant de l'histoire de la chirurgie berlinoise, et qui émit en revanche quelques doutes quant à la véracité de cette histoire. Il précisa que l'opération de Block avait eu lieu à Danzig et qu'il était peu probable qu'on trouvât encore des documents s'y rapportant. Meade suggéra également que l'absence de signes de tuberculose à l'autopsie pouvait s'expliquer par le fait qu'en réséquant les deux apex pulmonaires Block avait tout enlevé! Mis à part son caractère mélodramatique cette histoire non vérifiable ne représente qu'un fait divers sur la route de la chirurgie thoracique.

Le chirurgien parisien Théodore Tuffier ${ }^{14}$ réussit la première résection pulmonaire segmentaire avec survie du patient en 1891, et un autre Allemand, Themistokles Gluck, pratiqua la première lobectomie pour tuberculose en 1907.

L'ère moderne s'ouvre en 1935 lorsque Freedlander ${ }^{15}$ suscita la discussion de cette approche en présentant un cas de lobectomie au congrès de l'American Association for Thoracic Surgery. La réception fut d'autant plus mitigée que Freedlander, très honnêtement, rapporta un échec. Cinq ans plus tard, en 1940, devant le même auditoire, Dolley et Jones ${ }^{16}$ présentèrent sept cas et dans la discussion cette fois-ci dix-huit chirurgiens rapportèrent leurs quelques cas isolés, cinquante en tout, dont un certain nombre de succès! A partir de là, la résection pour tuberculose deviendra la routine quotidienne pour les chirurgiens thoraciques. Au début des années cinquante des milliers d'opérations avec un succès croissant furent publiées par Overholt aux EtatsUnis, Bérard en France, Brunner et moi-même en Suisse.

Brock en Angleterre et Harken et Bailey aux Etats-Unis avaient fait leurs premières armes avec la thoracoplastie dans les salles d'opération improvisées des sanatoria avant de développer une technique de plus en plus méticuleuse de résection pulmonaire, et d'acquérir une grande expérience de soins postopératoires. Je rappelle ici la contribution importante de ces chirurgiens à la médecine thoracique. En 1947, Brock publia une belle étude, dorénavant classique, de l'anatomie détaillée de l'arbre bronchique ${ }^{17}$, et en 1945 Bailey avait publié un livre sur le management pré- et postopératoire du patient thoracique ${ }^{18}$. De plus, les jeunes chirurgiens appelés sous les drapeaux en 1939 se virent rapidement confrontés à des cas de blessures du cœur et des grands vaisseaux. L'un d'eux, Dwight Harken, réussit l'exploit d'enlever 134 projectiles logés autour et dans le cœur et les grands vaisseaux 
sans perdre un seul des G.I. blessés. Lorsqu'il présenta ces cas pour la première fois à un staff meeting en présence d'Edward D. Churchill, médecinchef du corps expéditionnaire américain en Méditerranée, Churchill demanda ironiquement : «Did any of them live?», et Harken de répondre avec l'aplomb qu'on lui connaissait: «All of them!» Cette expérience de guerre le prédestinait à la chirurgie cardiaque et, de retour à Boston, il deviendra un des initiateurs de la chirurgie à cœur fermé, une chirurgie à nos yeux évidemment encore bien primitive, qui se développera cependant à pas de géant pour devenir en moins de cinquante ans la chirurgie cardiaque sophistiquée d'aujourd'hui.

Ayant vécu toute cette évolution depuis 1941, je peux dire que la tuberculose pulmonaire a réellement été le point de départ d'une évolution spectaculaire et qu'elle a été la base d'une discipline chirurgicale nouvelle, la chirurgie thoracique et cardiovasculaire! 


\section{Notes}

1 Naef A. P., Chirurgie thoracique, Ed. Médecine et Hygiène, Genève, 1988.

2 Naef A.P., The Story of Thoracic Surgery, Hogrefe + Huber Publisher, Toronto 1990.

3 Laennec R., L'auscultation médiate, Paris 1819.

4 Spengler L., Über Lungenkollapstherapie, in Corr. Blatt Schweiz. Ärzte 43, 1913, p. 1025.

5 Forlanini G., Primo caso di tisi pulmonare curato col pneumotorace artificiale, in Gazz. degli Osped. 68, 1882, p. 537.

6 Mann Thomas, Der Zauberberg.

7 Monaldi V., A propos du procédé d'aspiration endovacitaire des cavernes, in Rev. Tuberculose 5,1939, p. 848.

8 de Cérenville E., De l'intervention opératoire dans les maladies du poumon, in Rev. méd. Suisse rom. 5, 1885, p. 441.

9 Brauer L., Das Ziel und die Abarten der extrapleuralen Thorakoplastik, in Beitr. Klin. Tbk 51, 1922, p. 319 .

10 Sauerbruch F., Die Chirurgie der Brustorgane, Berlin, 1920 (2 éd.).

11 Overholt R.H., The Technique of Pulmonary Resection, C. Thomas, Publ., Springfield, Ill., 1949.

12 Walton G. L., Letter from Berlin, in Boston Med. and Surg. Journal 108, 1883, p. 262.

13 Meade R., History of Thoracic Surgery, Springfield 1961.

14 Tuffier T., Résection du poumon, in Sem. Méd., Paris 2, 1891, p. 202.

15 Freedlander S. O., Lobectomy, in Tbc. J.Th. Surgery 5, 1935, p. 132.

16 Dolley F.S. et al., Lobectomy, in J.Th. Surgery 10, 1940, p. 102.

17 Brock R.G., The Anatomy of the Broncheal Tree, Oxford University Press, London, 1947.

18 Bailey C.P., Diagnosis and Management of the Thoracic Patient, Y.B. Lippincott, Publ., Philadelphia, 1945. 


\section{Summary}

\section{Tuberculosis, the starting-point of thoracic surgery}

The amazing development of thoracic surgery was due to the extraordinary number of operations necessary in the treatment of diseases of epidemic proportions: tuberculosis, rheumatic heart disease and coronary disease. From 1939 onwards, the young chest surgeons were confronted with war wounds of the heart and great vessels. Back in civilian practice, they became the pioneers of an initially primitive, blind cardiac surgery. In the course of 40 years this surgery developed to become the highly specialized interdisciplinary specialty of today's cardiovascular surgery, the crowning of which is the routine heart transplantation of 1990.

\section{Zusammenfassung}

\section{Die Tuberkulose, Ausgangspunkt der Thoraxchirurgie}

Der gewaltige Aufschwung der Thoraxchirurgie im Lauf der letzten vierzig Jahre - vom Autor miterlebt - entspricht dem grossen Bedürfnis nach chirurgischen Behandlungsmethoden für weitverbreitete Krankheiten: Tuberkulose, Endocarditis rheumatica, Koronarsklerose. Am Anfang stand, schon seit der Jahrhundertwende, die Kollapsbehandlung der Tuberkulose. Der zweite Weltkrieg forderte die jungen Chirurgen, die sich in den Sanatorien geübt hatten, heraus, Verletzungen des Herzens und der grossen Gefässe chirurgisch zu versorgen. Sie wurden in der Nachkriegszeit die Pioniere der Herzchirurgie, die sich seither zu einem hochdifferenzierten, pluridisziplinären Fach entwickelt hat.

Prof. Dr.med.A.P. Naef

12 , av. Villardin

CH-1009 Pully 